\title{
On weighted integrability of functions defined by trigonometric series with $p$-bounded variation coefficients
}

\section{Bogdan Szal ${ }^{1 *}$ and Maciej Kubiak}

Correspondence:

B.Szal@wmie.uz.zgora.pl

1 Faculty of Mathematics, Computer

Science and Econometrics,

University of Zielona Góra, Zielona

Góra, Poland

\begin{abstract}
In this paper we introduce new classes of $p$-bounded variation sequences and give a sufficient and necessary condition for weighted integrability of trigonometric series with coefficients belonging to these classes. This is a generalization of the results obtained by the first author [J. Inequal. Appl. 2010:1-19, 2010] and Dyachenko and Tikhonov [Stud. Math. 193(3):285-306, 2009].
\end{abstract}

MSC: $42 \mathrm{~A} 16 ; 42 \mathrm{~A} 20 ; 42 \mathrm{~A} 32 ; 41 \mathrm{~A} 50$

Keywords: Trigonometric series; Sequences of $p$-bounded variation; Weighted $L^{p}$ integrability

\section{Introduction}

Let $L^{s}, 1 \leq s<\infty$, be the space of all $s$-power integrable functions $f$ of period $2 \pi$ with the norm

$$
\|f\|_{L^{s}}=\left(\int_{-\pi}^{\pi}|f(x)|^{s} d x\right)^{\frac{1}{s}}
$$

Write

$$
f(x)=\sum_{k=1}^{\infty} a_{k} \cos k x, \quad g(x)=\sum_{k=1}^{\infty} a_{k} \sin k x
$$

for those $x$, where the above series converge.

Denote by $\phi$ and $\lambda_{n}$ either $f$ or $g$ and either $a_{n}$ and $b_{n}$, respectively.

Let $\triangle_{r} a_{n}=a_{n}-a_{n+r}$ for a sequence of complex numbers $\left(a_{n}\right)$ and $r \in \mathbb{N}$.

Theorem 1 Let a nonnegative sequence $\left(\lambda_{n}\right) \in \Re, 1<s<\infty$ and $1-s<\alpha<1$. Then

$$
x^{-\alpha}|\phi|^{s} \in L^{1} \Longleftrightarrow \sum_{n=1}^{\infty} n^{\alpha+s-2} \lambda_{n}^{s}<\infty .
$$

\section{Springer}

(c) The Author(s) 2019. This article is distributed under the terms of the Creative Commons Attribution 4.0 International License (http://creativecommons.org/licenses/by/4.0/), which permits unrestricted use, distribution, and reproduction in any medium, provided you give appropriate credit to the original author(s) and the source, provide a link to the Creative Commons license, and indicate if changes were made. 
This theorem was proved for $\Re=D S$, where $D S$ denotes all decreasing sequences, in [1, $5,14]$, and [2]. Later, Theorem 1 was showed in [7] for

$$
\Re=\overline{G M}\left({ }_{1} \beta\right):=\left\{\left(a_{n}\right) \subset \mathbb{C}: \sum_{k=n}^{\infty}\left|\Delta_{1} a_{k}\right| \leq C \cdot{ }_{1} \beta_{n}\right\},
$$

and in [12] for

$$
\Re=G M\left({ }_{1} \beta\right):=\left\{\left(a_{n}\right) \subset \mathbb{C}: \sum_{k=n}^{2 n-1}\left|\Delta_{1} a_{k}\right| \leq C \cdot{ }_{1} \beta_{n}\right\},
$$

where ${ }_{1} \beta_{n}=\left|a_{n}\right| ; C$ here and throughout the paper denotes a positive constant.

The proof in the case of class

$$
\Re=G M\left({ }_{2} \beta\right):=\left\{\left(a_{n}\right) \subset \mathbb{C}: \sum_{k=n}^{2 n-1}\left|\Delta_{1} a_{k}\right| \leq C \cdot{ }_{2} \beta_{n}\right\},
$$

where ${ }_{2} \beta_{n}=\sum_{k=[n / c]}^{[c n]} \frac{\left|a_{k}\right|}{k}$ for some $c>1$, is included in [13].

In [3] Dyachenko and Tikhonov extended this theorem to the class

$$
\Re=\overline{G M}\left({ }_{3} \beta(\theta)\right):=\left\{\left(a_{n}\right) \subset \mathbb{C}: \sum_{k=n}^{\infty}\left|\Delta_{1} a_{k}\right| \leq C \cdot{ }_{3} \beta_{n}(\theta)\right\},
$$

where ${ }_{3} \beta_{n}(\theta)=n^{\theta-1} \sum_{k=[n / c]}^{\infty} \frac{\left|a_{k}\right|}{k^{\theta}}<\infty$ for some $c>1$ and $\theta \in(0,1]$.

From the articles of Dyachenko and Tikhonov [3] and Leindler [7], it is well known that

$$
\begin{aligned}
D S & \varsubsetneqq \overline{G M}\left({ }_{1} \beta\right) \varsubsetneqq G M\left({ }_{1} \beta\right) \varsubsetneqq G M\left({ }_{2} \beta\right) \\
& \varsubsetneqq \overline{G M}\left({ }_{3} \beta(1)\right) \subseteq \overline{G M}\left({ }_{3} \beta\left(\theta_{2}\right)\right) \subseteq \overline{G M}\left({ }_{3} \beta\left(\theta_{1}\right)\right),
\end{aligned}
$$

for $0<\theta_{1} \leq \theta_{2} \leq 1$.

Further, Szal defined a new class of sequences in the following way (see [9]):

Definition 1 Let $\beta:=\left(\beta_{n}\right)$ be a nonnegative sequence and $r$ a natural number. The sequence of complex numbers $a:=\left(a_{n}\right) \in \overline{G M}(\beta, r)$ if the relation

$$
\sum_{k=n}^{\infty}\left|\triangle_{r} a_{n}\right| \leq C \beta_{n}
$$

holds for all $n \in \mathbb{N}$.

Moreover, from [9] we know that

$$
\overline{G M}\left({ }_{3} \beta(\theta), r_{1}\right) \subsetneq \overline{G M}\left({ }_{3} \beta(\theta), r_{2}\right),
$$

where $r_{1}<r_{2}, \theta \in(0,1]$ and $r_{1} \mid r_{2}$. 
Let $r \in \mathbb{N}$ and $\alpha \in \mathbb{R}$. We define on the interval $[-\pi, \pi]$ an even function $\omega_{\alpha, r}$, which is given on the interval $[0, \pi]$ by the formula

$$
\omega_{\alpha, r}(x):=\left\{\begin{array}{l}
\left(x-\frac{2 l \pi}{r}\right)^{-\alpha} \quad \text { for } x \in\left(\frac{2 l \pi}{r}, \frac{(2 l+1) \pi}{r}\right] \text { and } l \in U_{1}, \\
\left(\frac{2(l+1) \pi}{r}-x\right)^{-\alpha} \quad \text { for } x \in\left(\frac{(2 l+1) \pi}{r}, \frac{2(l+1) \pi}{r}\right) \text { and } l \in U_{2}, \\
0 \quad \text { for } x=\frac{2 l \pi}{r} \text { and } l \in U_{3},
\end{array}\right.
$$

where $U_{1}=\{0,1, \ldots,[r / 2]\}$ if $r$ is an odd number and $U_{1}=\{0,1, \ldots,[r / 2]-1\}$ if $r$ is an even number, $U_{2}=\{0,1, \ldots,[r / 2]-1\}$ for $r \geq 2$, and $U_{3}=\{0,1, \ldots,[r / 2]\}$ for $r \geq 1$.

Theorem 1 was generalized for the class $\overline{G M}\left({ }_{3} \beta(\theta), r\right)$, where $r \in \mathbb{N}$ and $\theta \in(0,1]$, in [9]. We can formulate this result in the following way.

Theorem 2 ( $\left[9\right.$, Theorem 5]) Let a nonnegative sequence $\left(\lambda_{n}\right) \in \overline{G M}\left({ }_{3} \beta(\theta)\right.$, r), where $r \in \mathbb{N}$, $\theta \in(0,1]$ and $1 \leq s<\infty$. If

$$
1-\theta s<\alpha<1,
$$

then $\omega_{\alpha, r}|\phi|^{s} \in L^{1}$ if and only if

$$
\sum_{n=1}^{\infty} n^{\alpha+s-2}\left|\lambda_{n}\right|^{s}<\infty .
$$

Now, we define new classes of sequences.

Definition 2 Let $\beta:=\left(\beta_{n}\right)$ be a nonnegative sequence, $p$ a positive real number, $r \in \mathbb{N}$. One says that a sequence $a=\left(a_{n}\right)$ of complex numbers belongs to $G M(p, \beta, r)$ if the relation

$$
\left(\sum_{k=n}^{2 n-1}\left|\triangle_{r} a_{k}\right|^{p}\right)^{\frac{1}{p}} \leq C \beta_{n}
$$

holds for all $n \in \mathbb{N}$.

Moreover, we say that a sequence $\left(a_{n}\right) \in \overline{G M}(p, \beta, r)$ if the relation

$$
\left(\sum_{k=n}^{\infty}\left|\triangle_{r} a_{k}\right|^{p}\right)^{\frac{1}{p}} \leq C \beta_{n}
$$

holds for all $n \in \mathbb{N}$.

The class $G M(p, \beta, 1)$ was defined by Tikhonov and Liflyand in [8].

In this paper we present some properties of the classes $\overline{G M}\left(p,{ }_{3} \beta(\theta), r\right)$ and $G M(p$, $\left.{ }_{3} \beta(\theta), r\right)$. Moreover, we will generalize Theorem 2 for the class $G M\left(p,{ }_{3} \beta(\theta), r\right)$ with $0<$ $\theta<\frac{1}{s}$ and $r \in \mathbb{N}$.

We will write $I_{1} \ll I_{2}$ if there exists a positive constant $C$ such that $I_{1} \leq C I_{2}$. 


\section{Main results}

We formulate our results as follows:

Theorem 3 Let $r \in \mathbb{N}, \theta \in(0,1)$, and $p$ be a positive real number. Then

$$
\begin{aligned}
& \overline{G M}\left(p,{ }_{3} \beta(\theta), r\right)=G M\left(p,{ }_{3} \beta(\theta), r\right) \quad \text { and } \\
& \overline{G M}\left(p,{ }_{3} \beta(1), r\right) \subseteq G M\left(p,{ }_{3} \beta(1), r\right) .
\end{aligned}
$$

Theorem 4 Let $r \in \mathbb{N}, \theta \in(0,1)$, and $p_{1}, p_{2}$ be two positive real numbers such that $0<$ $p_{1}<p_{2}$. Then

$$
G M\left(p_{1},{ }_{3} \beta(\theta), r\right) \varsubsetneqq G M\left(p_{2},{ }_{3} \beta(\theta), r\right) .
$$

Theorem 5 Let $r_{1}, r_{2} \in \mathbb{N}, r_{1}<r_{2}, \theta \in(0,1]$ and $p \geq 1$. If $r_{1} \mid r_{2}$, then

$$
\operatorname{GM}\left(p,{ }_{3} \beta(\theta), r_{1}\right) \varsubsetneqq G M\left(p,{ }_{3} \beta(\theta), r_{2}\right) .
$$

Theorem 6 Let $\left(b_{n}\right) \in G M\left(p,{ }_{3} \beta(\theta), r\right)$, where $r \in \mathbb{N}, p \geq 1,0<\theta<\frac{1}{p}$ and $1 \leq s<\infty$. If

$$
1-\theta s-s+\frac{s}{p}<\alpha<1
$$

and

$$
\sum_{n=1}^{\infty} n^{\alpha-2-\frac{s}{p}+2 s}\left|b_{n}\right|^{s}<\infty
$$

then $\omega_{\alpha . r}|\phi|^{s} \in L^{1}$.

Theorem 7 Let a nonnegative sequence $\left(b_{n}\right)$ belong to $G M\left(p,{ }_{3} \beta(\theta), r\right)$, where $r \in \mathbb{N}, p \geq 1$, $0<\theta<\frac{1}{p}$ and $1 \leq s<\infty$. If

$$
1-\theta s<\alpha<1+s
$$

and $\omega_{\alpha . r}|\phi|^{s} \in L^{1}$ then

$$
\sum_{n=1}^{\infty} n^{\alpha-2+\frac{s}{p}} b_{n}^{s}<\infty
$$

Remark 1 If we take $p=1$, then the result of Szal [9] (Theorem 2) follows from our Theorem 6 and 7. Moreover, by the embedding relations (1) and (2), we can also derive from Theorem 6 and 7 the result of Dyachenko and Tikhonov [3] and all the results mentioned before. 


\section{Auxiliary results}

For $n \in \mathbb{N}$ and $k=0,1,2, \ldots$, denote by

$$
\begin{aligned}
& D_{k, r}(x)=\frac{\sin (k+r / 2) x}{2 \sin (r x / 2)}, \\
& \widetilde{D}_{k, r}(x)=\frac{\cos (k+r / 2) x}{2 \sin (r x / 2)}
\end{aligned}
$$

the Dirichlet-type kernels.

Lemma 1 ([10, Lemma 3.1] and [11, Lemma 17]) Let $r \in \mathbb{N}, l \in \mathbb{Z}$, and $\left(a_{n}\right) \subset \mathbb{C}$. If $x \neq \frac{2 l \pi}{r}$, then for all $m \geq n$

$$
\begin{aligned}
& \sum_{k=n}^{m} a_{k} \cos k x=\sum_{k=n}^{m} \triangle_{r} a_{k} D_{k, r}(x)-\sum_{k=m+1}^{m+r} a_{k} D_{k,-r}(x)+\sum_{k=n}^{n+r-1} a_{k} D_{k,-r}(x), \\
& \sum_{k=n}^{m} a_{k} \sin k x=\sum_{k=m+1}^{m+r} a_{k} \widetilde{D}_{k,-r}(x)-\sum_{k=n}^{n+r-1} a_{k} \widetilde{D}_{k,-r}(x)-\sum_{k=n}^{m} \triangle_{r} a_{k} \widetilde{D}_{k, r}(x) .
\end{aligned}
$$

Lemma $2([6$, Corollary 1$])$ Let $p \geq 1, \gamma_{n}>0$, and $a_{n} \geq 0$ for $n \in \mathbb{N}$. Then

$$
\begin{aligned}
& \sum_{n=1}^{\infty} \gamma_{n}\left(\sum_{k=1}^{n} a_{k}\right)^{p} \leq p^{p} \sum_{n=1}^{\infty} \gamma_{n}^{1-p} a_{n}^{p}\left(\sum_{k=n}^{\infty} \gamma_{k}\right)^{p}, \\
& \sum_{n=1}^{\infty} \gamma_{n}\left(\sum_{k=n}^{\infty} a_{k}\right)^{p} \leq p^{p} \sum_{n=1}^{\infty} \gamma_{n}^{1-p} a_{n}^{p}\left(\sum_{k=1}^{n} \gamma_{k}\right)^{p} .
\end{aligned}
$$

Lemma 3 ([4, Theorem 19]) If $a_{n} \geq 0$ for $n \in \mathbb{N}$ and $0<p_{1} \leq p_{2}<\infty$, then

$$
\left(\sum_{n=1}^{\infty} a_{n}^{p_{2}}\right)^{\frac{1}{p_{2}}} \leq\left(\sum_{n=1}^{\infty} a_{n}^{p_{1}}\right)^{\frac{1}{p_{1}}}
$$

Lemma 4 ([4]) Let $a_{k} \geq 0$ for $k \in \mathbb{N}$ and $p \geq 1$. Then

$$
\left(\frac{1}{n} \sum_{k=n}^{2 n-1} a_{k}^{p}\right)^{\frac{1}{p}} \geq \frac{1}{n} \sum_{k=n}^{2 n-1} a_{k}
$$

Lemma 5 Let $\left(a_{k}\right) \subset \mathbb{C}, p \geq 1, r, n \in \mathbb{N}$ and $d \in \mathbb{N}_{0}=\mathbb{N} \cup\{0\}$. Then

$$
\sum_{k=2^{d+1}(n+1)}^{2^{d+1}(n+1)+r-1}\left|a_{k}\right| \leq \sum_{k=2^{d}(n+1)}^{2^{d}(n+1)+r-1}\left|a_{k}\right|+\left[2^{d}(n+1)\right]^{1-\frac{1}{p}}\left(\sum_{k=2^{d}(n+1)}^{2^{d+1}(n+1)-1}\left|\Delta_{r} a_{k}\right|^{p}\right)^{\frac{1}{p}} .
$$


Proof From Lemma 4 we have

$$
\begin{aligned}
\left(\sum_{k=2^{d}(n+1)}^{2^{d+1}(n+1)-1}\left|\triangle_{r} a_{k}\right|^{p}\right)^{\frac{1}{p}} & =\left[2^{d}(n+1)\right]^{\frac{1}{p}}\left(\frac{1}{2^{d}(n+1)} \sum_{k=2^{d}(n+1)}^{2^{d+1}(n+1)-1}\left|\triangle_{r} a_{k}\right|^{p}\right)^{\frac{1}{p}} \\
& \geq\left[2^{d}(n+1)\right]^{\frac{1}{p}} \frac{1}{2^{d}(n+1)} \sum_{k=2^{d}(n+1)}^{2^{d+1}(n+1)-1}\left|\triangle_{r} a_{k}\right| \\
& \geq\left[2^{d}(n+1)\right]^{\frac{1}{p}-1}\left(\sum_{k=2^{d+1}(n+1)}^{2^{d+1}(n+1)+r-1}\left|a_{k}\right|-\sum_{k=2^{d}(n+1)}^{2^{d}(n+1)+r-1}\left|a_{k}\right|\right) .
\end{aligned}
$$

Hence

$$
\sum_{k=2^{d+1}(n+1)}^{2^{d+1}(n+1)+r-1}\left|a_{k}\right| \leq \sum_{k=2^{d}(n+1)}^{2^{d}(n+1)+r-1}\left|a_{k}\right|+\left[2^{d}(n+1)\right]^{1-\frac{1}{p}}\left(\sum_{k=2^{d}(n+1)}^{2^{d+1}(n+1)-1}\left|\triangle_{r} a_{k}\right|^{p}\right)^{\frac{1}{p}}
$$

and this ends our proof.

Lemma 6 Let $\left(a_{k}\right) \in G M\left(p,{ }_{3} \beta(\theta), r\right), p \geq 1, r \in \mathbb{N}, d \in \mathbb{N}_{0}$, and $0<\theta<\frac{1}{p}$. Then

$$
\sum_{k=2^{d}(n+1)}^{2^{d}(n+1)+r-1}\left|a_{k}\right| \leq C \frac{1}{1-2^{\theta-\frac{1}{p}}}\left(2^{d}(n+1)\right)^{\theta-\frac{1}{p}} \sum_{k=\left[\frac{2^{d(n+1)}}{c}\right]}^{\infty} \frac{\left|a_{k}\right|}{k^{\theta}}
$$

Proof We have

$$
\sum_{k=2^{d}(n+1)}^{2^{d}(n+1)+r-1}\left|a_{k}\right| \leq \sum_{j=0}^{\infty} \sum_{k=2^{2} 2^{d}(n+1)}^{2^{j+1} 2^{d}(n+1)-1}\left|\triangle_{r} a_{k}\right|
$$

Using Hölder inequality with $p>1$, we get

$$
\begin{aligned}
& \sum_{j=0}^{\infty} \sum_{k=2^{2} 2^{d}(n+1)}^{2^{j+1} 2^{d}(n+1)-1}\left|\triangle_{r} a_{k}\right| \\
& \leq \sum_{j=0}^{\infty}\left[\left(\sum_{k=2^{j} 2^{d}(n+1)}^{2^{j+1} 2^{d}(n+1)-1}\left|\triangle_{r} a_{k}\right|^{p}\right)^{\frac{1}{p}}\left(\sum_{k=2^{j} 2^{d}(n+1)}^{2^{j+1} 2^{d}(n+1)-1} 1^{\frac{p}{p-1}}\right)^{1-\frac{1}{p}}\right] \\
& \leq C \sum_{j=0}^{\infty}\left(2^{j} 2^{d}(n+1)\right)^{1-\frac{1}{p}}\left(2^{j} 2^{d}(n+1)\right)^{\theta-1} \sum_{k=\left[\frac{j_{2} 2_{(n+1)}}{c}\right]}^{\infty} \frac{\left|a_{k}\right|}{k^{\theta}} \\
& \leq C\left(2^{d}(n+1)\right)^{\theta-\frac{1}{p}} \sum_{k=\left[\frac{2^{d}(n+1)}{c}\right]}^{\infty} \frac{\left|a_{k}\right|}{k^{\theta}} \sum_{j=0}^{\infty}\left(2^{\theta-\frac{1}{p}}\right)^{j} .
\end{aligned}
$$


When $p=1$, we have

$$
\begin{aligned}
\sum_{j=0}^{\infty} \sum_{k=2^{2} 2^{d}(n+1)}^{2^{j+1} 2^{d}(n+1)-1}\left|\triangle_{r} a_{k}\right| & \leq C \sum_{j=0}^{\infty}\left(2^{j} 2^{d}(n+1)\right)^{\theta-1} \sum_{k=\left[\frac{2^{j} 2^{d}(n+1)}{c}\right]}^{\infty} \frac{\left|a_{k}\right|}{k^{\theta}} \\
& \leq C\left(2^{d}(n+1)\right)^{\theta-1} \sum_{k=\left[\frac{2^{d}(n+1)}{c}\right]}^{\infty} \frac{\left|a_{k}\right|}{k^{\theta}} \sum_{j=0}^{\infty}\left(2^{\theta-1}\right)^{j} .
\end{aligned}
$$

If $\theta-\frac{1}{p}<0$, then

$$
\sum_{k=2^{d}(n+1)}^{2^{d}(n+1)+r-1}\left|a_{k}\right| \leq C \frac{1}{1-2^{\theta-\frac{1}{p}}}\left(2^{d}(n+1)\right)^{\theta-\frac{1}{p}} \sum_{k=\left[\frac{2^{d}(n+1)}{c}\right]}^{\infty} \frac{\left|a_{k}\right|}{k^{\theta}}
$$

and our proof is complete.

\section{Proofs}

\subsection{Proof of Theorem 3}

Let $\left(a_{n}\right) \in G M\left(p,{ }_{3} \beta(\theta), r\right)$, where $p>0, r \in \mathbb{N}$, and $\theta \in(0,1)$. Then

$$
\begin{aligned}
\left(\sum_{k=n}^{\infty}\left|\triangle_{r} a_{k}\right|^{p}\right)^{\frac{1}{p}} & =\left(\sum_{d=0}^{\infty} \sum_{k=2^{d_{n}}}^{2^{d+1} n-1}\left|\triangle_{r} a_{k}\right|^{p}\right)^{\frac{1}{p}} \\
& \leq\left(\sum_{d=0}^{\infty}\left(C\left(2^{d} n\right)^{\theta-1} \sum_{k=\left[\frac{2^{d} d_{n}}{c}\right]}^{\infty} \frac{\left|a_{k}\right|}{k^{\theta}}\right)^{p}\right)^{\frac{1}{p}} \\
& \leq C n^{\theta-1} \sum_{k=\left[\frac{n}{c}\right]}^{\infty} \frac{\left|a_{k}\right|}{k^{\theta}}\left(\sum_{d=0}^{\infty}\left(2^{(\theta-1) p}\right)^{d}\right)^{\frac{1}{p}}
\end{aligned}
$$

If $0<\theta<1$ then $(\theta-1) p<0$, and we have

$$
\left(\sum_{k=n}^{\infty}\left|\triangle_{r} a_{k}\right|^{p}\right)^{\frac{1}{p}} \leq C\left(\frac{1}{1-2^{(\theta-1) p}}\right)^{\frac{1}{p}} n^{\theta-1} \sum_{k=\left[\frac{n}{c}\right]}^{\infty} \frac{\left|a_{k}\right|}{k^{\theta}}
$$

So $\left(a_{n}\right) \in \overline{G M}\left(p,{ }_{3} \beta(\theta), r\right)$.

Now we assume $\left(a_{n}\right) \in \overline{G M}\left(p,{ }_{3} \beta(1), r\right), p>0, r \in \mathbb{N}$. We have

$$
\left(\sum_{k=n}^{2 n-1}\left|\triangle_{r} a_{k}\right|^{p}\right)^{\frac{1}{p}} \leq\left(\sum_{k=n}^{\infty}\left|\triangle_{r} a_{k}\right|^{p}\right)^{\frac{1}{p}} \leq C n^{\theta-1} \sum_{k=\left[\frac{n}{c}\right]}^{\infty} \frac{\left|a_{k}\right|}{k^{\theta}} .
$$

This means $\left(a_{n}\right) \in G M\left(p,{ }_{3} \beta(1), r\right)$. 


\subsection{Proof of Theorem 4}

Let $r \in \mathbb{N}, \theta \in(0,1], 0<p_{1} \leq p_{2}$, and $\left(a_{n}\right) \in G M\left(p_{1},{ }_{3} \beta(\theta), r\right)$. We will show that $G M\left(p_{1},{ }_{3} \beta(\theta), r\right) \subseteq G M\left(p_{2},{ }_{3} \beta(\theta), r\right)$. Using Lemma 3, we have

$$
\left(\sum_{k=n}^{2 n-1}\left|\triangle_{r} a_{k}\right|^{p_{2}}\right)^{\frac{1}{p_{2}}} \leq\left(\sum_{k=n}^{2 n-1}\left|\triangle_{r} a_{k}\right|^{p_{1}}\right)^{\frac{1}{p_{1}}} \leq c n^{\theta-1} \sum_{k=n}^{\infty} \frac{\left|a_{k}\right|}{k^{\theta}} .
$$

This means that $\left(a_{n}\right) \in G M\left(p_{2},{ }_{3} \beta(\theta), r\right)$.

Now we will show that $G M\left(p_{1}, 3 \beta(\theta), r\right) \neq G M\left(p_{2}, 3 \beta(\theta), r\right)$ for $0<p_{1}<p_{2}$. Let

$$
a_{n}=\left\{\begin{array}{l}
\frac{1}{n^{2}}, \quad \text { when } 2 r \nmid n, \\
\frac{1}{(n-r)^{2}}+\frac{1}{n^{2} n^{\frac{1}{p_{2}}}}, \quad \text { when } 2 r \mid n
\end{array}\right.
$$

We prove that $\left(a_{n}\right) \in G M\left(p_{2},{ }_{3} \beta(\theta), r\right)$. Suppose

$$
\begin{aligned}
& A_{n}=\{k \in \mathbb{N}: n \leq k \leq 2 n-1 \text { and } 2 r \mid k\}, \\
& B_{n}=\{k \in \mathbb{N}: n \leq k \leq 2 n-1,2 r \nmid k \text { and } 2 r \nmid k+r\}, \\
& C_{n}=\{k \in \mathbb{N}: n \leq k \leq 2 n-1,2 r \nmid k \text { and } 2 r \mid k+r\} .
\end{aligned}
$$

Then

$$
\begin{aligned}
& \left(\sum_{k=n}^{2 n-1}\left|a_{k}-a_{k+r}\right|^{p_{2}}\right)^{\frac{1}{p_{2}}} \\
& =\left(\sum_{k \in A_{n}}\left|\frac{1}{(k-r)^{2}}+\frac{1}{k^{2} k^{\frac{1}{p_{2}}}}-\frac{1}{(k+r)^{2}}\right|^{p_{2}}\right. \\
& \left.\left.\left.\quad+\sum_{k \in B_{n}}\left|\frac{1}{k^{2}}-\frac{1}{(k+r)^{2}}\right|^{p_{2}}+\sum_{k \in C_{n}}\left|\frac{1}{k^{2}}-\frac{1}{k^{2}}-\frac{1}{(k+r)^{2}(k+r)^{\frac{1}{p_{2}}}}\right|^{p_{2}}\right)^{\frac{1}{p_{2}}}\right)^{p_{2}}\right)^{\frac{1}{p_{2}}} \\
& \leq\left(\sum_{k \in A_{n}}\left(\frac{4 k r}{\frac{1}{4} k^{2} k^{2}}+\frac{1}{k^{2+\frac{1}{p_{2}}}}\right)^{p_{2}}+\sum_{k \in B_{n}}\left(\frac{2 k r+r^{2}}{k^{2}(k+r)^{2}}\right)^{p_{2}}+\sum_{k \in C_{n}}\left(\frac{1}{(k+r)^{2+\frac{1}{p_{2}}}}\right)^{2 n-1}\left(\frac{1}{\sum^{2}}\right)^{p_{2}}\right)^{\frac{1}{p_{2}}} \leq \frac{17 r}{n^{2}} .
\end{aligned}
$$

Moreover,

$$
\frac{17 r}{n^{2}} \leq 2^{2+\theta} 17 r\left(n^{\theta-1} \sum_{k=n}^{2 n-1} \frac{1}{k^{2}} \frac{1}{k^{\theta}}\right) \leq 2^{2+\theta} 17 r n^{\theta-1} \sum_{k=\left[\frac{n}{c}\right]}^{\infty} \frac{\left|a_{k}\right|}{k^{\theta}} .
$$

This means $\left(a_{n}\right) \in G M\left(p_{2},{ }_{3} \beta(\theta), r\right)$. We will show that $\left(a_{n}\right) \notin G M\left(p_{1},{ }_{3} \beta(\theta), r\right)$. We have

$$
\left(\sum_{k=n}^{2 n-1}\left|a_{k}-a_{k+r}\right|^{p_{1}}\right)^{\frac{1}{p_{1}}} \geq\left(\sum_{k \in C_{n}} \frac{1}{(k+r)^{2 p_{1}+\frac{p_{1}}{p_{2}}}}\right)^{\frac{1}{p_{1}}} \geq \frac{1}{(4 r)^{2+\frac{1}{p_{2}}+\frac{2}{p_{1}}}} \frac{n^{\frac{1}{p_{1}}}}{n^{2+\frac{1}{p_{2}}}} .
$$


Let

$$
\begin{aligned}
& D_{n}=\left\{k \in \mathbb{N}:\left[\frac{n}{c}\right] \leq k \text { and } 2 r \mid k\right\}, \\
& E_{n}=\left\{k \in \mathbb{N}:\left[\frac{n}{c}\right] \leq k \text { and } 2 r \nmid k\right\} .
\end{aligned}
$$

On the other hand, we get

$$
\begin{aligned}
n^{\theta-1} \sum_{k=\left[\frac{n}{c}\right]}^{\infty} \frac{a_{k}}{k^{\theta}} & =n^{\theta-1}\left(\sum_{k \in D_{n}} \frac{1}{k^{2} k^{\theta}}+\sum_{k \in E_{n}}\left(\frac{1}{(k-r)^{2}}+\frac{1}{k^{2+\frac{1}{p_{2}}}}\right) \frac{1}{k^{\theta}}\right) \\
& \leq 5 n^{\theta-1} \sum_{k=\left[\frac{n}{c}\right]}^{\infty} \frac{1}{k^{2+\theta}} \ll n^{-2} .
\end{aligned}
$$

Therefore the inequality

$$
\left(\sum_{k=n}^{2 n-1}\left|\triangle_{r} a_{k}\right|^{p_{1}}\right)^{\frac{1}{p_{1}}} \leq C n^{\theta-1} \sum_{k=\left[\frac{n}{c}\right]}^{\infty} \frac{a_{k}}{k^{\theta}}
$$

cannot be satisfied because $n^{\frac{1}{p_{1}}-\frac{1}{p_{2}}} \rightarrow \infty$ as $n \rightarrow \infty$.

\subsection{Proof of Theorem 5}

Let $r_{1}, r_{2} \in \mathbb{N}, r_{1} \leq r_{2}, r_{1} \mid r_{2}, p \geq 1$ and $\left(a_{n}\right) \in G M\left(p,{ }_{3} \beta(\theta), r_{1}\right)$.

If $r_{1} \mid r_{2}$, then $r_{2}=\alpha r_{1}$, where $\alpha \in \mathbb{N}$. Using Hölder inequality with $p>1$, we have

$$
\begin{aligned}
& \left(\sum_{k=n}^{2 n-1}\left|a_{k}-a_{k+r_{2}}\right|^{p}\right)^{\frac{1}{p}} \\
& =\left(\sum_{k=n}^{2 n-1}\left|\sum_{l=0}^{\alpha-1}\left(a_{k+l r_{1}}-a_{\left.k+(l+1) r_{1}\right)}\right)\right|^{p}\right)^{\frac{1}{p}} \\
& \leq\left(\sum_{k=n}^{2 n-1}\left(\sum_{l=0}^{\alpha-1}\left|a_{k+l r_{1}}-a_{k+(l+1) r_{1}}\right|\right)^{p}\right)^{\frac{1}{p}} \\
& \leq\left(\sum_{k=n}^{2 n-1}\left(\left(\sum_{l=0}^{\alpha-1}\left|a_{k+l r_{1}}-a_{k+(l+1) r_{1}}\right|^{p}\right)^{\frac{1}{p}}\left(\sum_{l=0}^{\alpha-1} \frac{p}{p^{p-1}}\right)^{1-\frac{1}{p}}\right)^{p}\right)^{\frac{1}{p}} \\
& \leq \alpha^{1-\frac{1}{p}}\left(\sum_{k=n}^{2 n-1}\left(\sum_{l=0}^{\alpha-1}\left|a_{k+l r_{1}}-a_{k+(l+1) r_{1}}\right|^{p}\right)\right)^{\frac{1}{p}} \\
& \leq \alpha^{1-\frac{1}{p}}\left(\sum_{l=0}^{\alpha-1}\left(C\left(n+l r_{1}\right)^{\theta-1} \sum_{k=\left[\frac{n+l r_{1}}{c}\right]}^{\infty} \frac{\left|a_{k}\right|}{k^{\theta}}\right)^{p}\right)^{\frac{1}{p}} \\
& \leq \alpha C n^{\theta-1} \sum_{k=\left[\frac{n}{c}\right]}^{\infty} \frac{\left|a_{k}\right|}{k^{\theta}} .
\end{aligned}
$$


If $p=1$ then

$$
\begin{aligned}
\sum_{k=n}^{2 n}\left|a_{k}-a_{k+r_{2}}\right| & \leq \sum_{k=n}^{2 n-1} \sum_{l=0}^{\alpha-1}\left|a_{k+l r_{1}}-a_{k+(l-1) r_{1}}\right| \\
& \leq C \sum_{l=0}^{\alpha-1}\left(n+l r_{1}\right)^{\theta-1} \sum_{k=\left[\frac{n+l r_{1}}{c}\right]}^{\infty} \frac{\left|a_{k}\right|}{k^{\theta}} \leq \alpha C n^{\theta-1} \sum_{k=\left[\frac{n}{c}\right]}^{\infty} \frac{\left|a_{k}\right|}{k^{\theta}}
\end{aligned}
$$

Hence $\left(a_{n}\right) \in \operatorname{GM}\left(p,{ }_{3} \beta(\theta), r_{2}\right)$.

Now, we will show that $\operatorname{GM}\left(p,{ }_{3} \beta(\theta), r_{1}\right) \varsubsetneqq G M\left(p,{ }_{3} \beta(\theta), r_{2}\right)$, when $r_{1}<r_{2}$. Let $a_{n}=\frac{2+\alpha_{n}}{n^{2}}$, where $\alpha_{n}= \begin{cases}-1, & \text { when } r_{1} \mid n, \\ 1, & \text { when } r_{1} \nmid n\end{cases}$

We will prove that $\left(a_{n}\right) \in G M\left(p,{ }_{3} \beta(\theta), r_{2}\right)$ and $\left(a_{n}\right) \notin G M\left(p,{ }_{3} \beta(\theta), r_{1}\right)$. Let

$$
\begin{aligned}
& A_{n}:=\left\{k \in \mathbb{N}: n \leq k \leq 2 n-1 \text { and } r_{2} \mid k\right\}, \\
& B_{n}:=\left\{k \in \mathbb{N}: n \leq k \leq 2 n-1 \text { and } r_{2} \nmid k\right\} .
\end{aligned}
$$

Then using Lemma 3 for $p \geq 1$, we have

$$
\begin{aligned}
\left(\sum_{k=n}^{2 n-1}\left|a_{k}-a_{k+r_{2}}\right|^{p}\right)^{\frac{1}{p}} & =\left(\left(\sum_{k \in A_{n}}+\sum_{k \in B_{n}}\right)\left|a_{k}-a_{k+r_{2}}\right|^{p}\right)^{\frac{1}{p}} \\
& =\left(\sum_{k \in A_{n}}\left|\frac{1}{k^{2}}-\frac{1}{\left(k+r_{2}\right)^{2}}\right|^{p}+\sum_{k \in B_{n}}\left|\frac{3}{k^{2}}-\frac{3}{\left(k+r_{2}\right)^{2}}\right|^{p}\right)^{\frac{1}{p}} \\
& \leq\left(3^{p} \sum_{k=n}^{2 n-1}\left|\frac{\left(k+r_{2}\right)^{2}-k^{2}}{\left(k+r_{2}\right)^{2} k^{2}}\right|^{p}\right)^{\frac{1}{p}} \\
& =3\left(\sum_{k=n}^{2 n-1}\left|\frac{2 r_{2} k+r_{2}^{2}}{\left(k+r_{2}\right)^{2} k^{2}}\right|^{p}\right)^{\frac{1}{p}} \\
& \leq 6 r_{2}\left(\sum_{k=n}^{2 n-1}\left(\frac{1}{k^{3}}\right)^{p}\right)^{\frac{1}{p}} \leq 6 r_{2} \sum_{k=n}^{2 n-1} \frac{1}{k^{3}} \leq \frac{6 r_{2}}{n} \sum_{k=n}^{2 n-1} \frac{1}{k^{2}} .
\end{aligned}
$$

Moreover,

$$
\begin{aligned}
\frac{6 r_{2}}{n} \sum_{k=n}^{2 n-1} \frac{1}{k^{2}} & =6 r_{2} n^{\theta-1} \frac{1}{(2 n)^{\theta}} 2^{\theta} \sum_{k=n}^{2 n-1} \frac{1}{k^{2}} \\
& \leq 6 r_{2} 2^{\theta} n^{\theta-1} \sum_{k=n}^{2 n-1} \frac{1}{k^{2}} \frac{1}{k^{\theta}} \\
& \leq 6 r_{2} 2^{\theta} n^{\theta-1} \sum_{k=n}^{2 n-1} \frac{a_{k}}{k^{\theta}} \leq 6 r_{2} 2^{\theta} n^{\theta-1} \sum_{k=\left[\frac{n}{c}\right]}^{\infty} \frac{a_{k}}{k^{\theta}}
\end{aligned}
$$


It means that $\left(a_{n}\right) \in G M\left(p,{ }_{3} \beta(\theta), r_{2}\right)$. Furthermore,

$$
\begin{aligned}
\left(\sum_{k=n}^{2 n-1}\left|a_{k}-a_{k+r_{1}}\right|^{p}\right)^{\frac{1}{p}} & \geq\left(\sum_{k \in A_{n}}\left|a_{k}-a_{k+r_{1}}\right|^{p}\right)^{\frac{1}{p}} \geq\left(\sum_{k \in A_{n}}\left|\frac{1}{k^{3}}-\frac{3}{\left(k+r_{1}\right)^{2}}\right|^{p}\right)^{\frac{1}{p}} \\
& =\left(\sum_{k \in A_{n}}\left|\frac{\left(k+r_{1}\right)^{2}-3 k^{2}}{\left(k+r_{1}\right)^{2} k^{2}}\right|^{p}\right)^{\frac{1}{p}}=\left(\sum_{k \in A_{n}}\left|\frac{-2 k^{2}+2 k r_{1}+r_{1}^{2}}{\left(k+r_{1}\right)^{2} k^{2}}\right|^{p}\right)^{\frac{1}{p}} .
\end{aligned}
$$

If $n \geq 5 r_{1}$, then $2 n^{2}-2 n r_{1}-r_{1}^{2} \geq\left(n+r_{1}\right)^{2}$. Whence for $n \geq 5 r_{1}$,

$$
\begin{aligned}
\left(\sum_{k=n}^{2 n-1}\left|a_{k}-a_{k+r_{1}}\right|^{p}\right)^{\frac{1}{p}} & \geq\left(\sum_{k \in A_{n}}\left(\frac{2 k^{2}-2 k r_{1}-r_{1}^{2}}{\left(k+r_{1}\right)^{2} k^{2}}\right)^{p}\right)^{\frac{1}{p}} \\
& \geq \frac{1}{(2 n)^{2}}\left(\frac{n}{2 r_{1}}\right)^{\frac{1}{p}}=\frac{1}{2^{2+\frac{1}{p}} r_{1}} n^{-2+\frac{1}{p}}
\end{aligned}
$$

On the other hand,

$$
n^{\theta-1} \sum_{k=\left[\frac{n}{c}\right]}^{\infty} \frac{a_{k}}{k^{\theta}} \leq n^{\theta-1} \sum_{k=\left[\frac{n}{c}\right]}^{\infty} \frac{3}{k^{2}} \frac{1}{k^{\theta}} \leq 3 n^{\theta-1} \sum_{k=\left[\frac{n}{c}\right]}^{\infty} \frac{1}{k^{2+\theta}} \ll n^{-2} .
$$

Therefore, the inequality

$$
\left(\sum_{k=n}^{2 n-1}\left|\triangle_{r_{1}} a_{k}\right|^{p}\right)^{\frac{1}{p}} \leq C n^{\theta-1} \sum_{k=\left[\frac{n}{c}\right]}^{\infty} \frac{a_{k}}{k^{\theta}}
$$

cannot be satisfied because $n^{\frac{1}{p}} \rightarrow \infty$ as $n \rightarrow \infty$.

\subsection{Proof of Theorem 6}

We prove the theorem for the case when $\phi(x)=g(x)$. We have

$$
\left\|\omega_{\alpha, r}|g|^{s}\right\|_{L^{1}}=2 \int_{0}^{\pi} \omega_{\alpha, r}(x)|g(x)|^{s} d x
$$

For an odd $r$,

$$
\begin{aligned}
\int_{0}^{\pi} \omega_{\alpha, r}(x)|g(x)|^{s} d x= & \sum_{l=0}^{[r / 2]} \int_{\frac{2 l \pi}{r}}^{\frac{2 l \pi}{r}+\frac{\pi}{r}} \omega_{\alpha, r}(x)\left|\sum_{k=1}^{\infty} b_{k} \sin k x\right|^{s} d x \\
& +\sum_{l=0}^{[r / 2]-1} \int_{\frac{2 l \pi}{r}+\frac{\pi}{r}}^{\frac{2(l+1) \pi}{r}} \omega_{\alpha, r}(x)\left|\sum_{k=1}^{\infty} b_{k} \sin k x\right|^{s} d x
\end{aligned}
$$

(for $r=1$ the last sum should be omitted), and for an even $r$,

$$
\int_{0}^{\pi} \omega_{\alpha, r}(x)|g(x)|^{s} d x=\sum_{l=0}^{[r / 2]}\left(\int_{\frac{2 l \pi}{r}}^{\frac{2 l \pi}{r}+\frac{\pi}{r}}+\int_{\frac{2 l \pi}{r}+\frac{\pi}{r}}^{\frac{2(l+1) \pi}{r}}\right) \omega_{\alpha, r}(x)\left|\sum_{k=1}^{\infty} b_{k} \sin k x\right|^{s} d x .
$$


Now, we estimate the following integral:

$$
\begin{aligned}
\int_{\frac{2 l \pi}{r}}^{\frac{2 l \pi}{r}+\frac{\pi}{r}} \omega_{\alpha, r}(x)\left|\sum_{k=1}^{\infty} b_{k} \sin k x\right|^{s} d x \ll & \left(\int_{\frac{2 l \pi}{r}}^{\frac{2 l \pi}{r}+\frac{\pi}{r}} \omega_{\alpha, r}(x)\left|\sum_{k=1}^{n} b_{k} \sin k x\right|^{s} d x\right. \\
& \left.+\int_{\frac{2 l \pi}{r}}^{\frac{2 l \pi}{r}+\frac{\pi}{r}} \omega_{\alpha, r}(x)\left|\sum_{k=n+1}^{\infty} b_{k} \sin k x\right|^{s} d x\right) \\
:= & I_{1}+I_{2} .
\end{aligned}
$$

By Lemma 2, for $\alpha<1$, we have

$$
\begin{aligned}
I_{1} & =\sum_{n=r}^{\infty} \int_{\frac{2 l \pi}{r}+\frac{\pi}{n+1}}^{\frac{2 l \pi}{r}+\frac{\pi}{n}}\left(x-\frac{2 l \pi}{r}\right)^{-\alpha}\left|\sum_{k=1}^{n} b_{k} \sin k x\right|^{s} d x \\
& \ll \sum_{n=r}^{\infty} n^{\alpha-2}\left(\sum_{k=1}^{n}\left|b_{k}\right|\right)^{s} \\
& \leq \sum_{n=1}^{\infty} n^{\alpha-2-\frac{s}{p}+2 s}\left|b_{n}\right|^{s} .
\end{aligned}
$$

Using Lemma 1 when $m \rightarrow \infty$ and the inequality

$$
\frac{r}{\pi} x-2 l \leq\left|\sin \frac{r x}{2}\right| \quad \text { for } x \in\left(\frac{2 l \pi}{r}, \frac{2 l \pi}{r}+\frac{\pi}{r}\right) \text {, }
$$

we get

$$
\begin{aligned}
& I_{2}=\sum_{n=r}^{\infty} \int_{2 l \pi / r+\pi /(n+1)}^{2 l \pi / r+\pi / n}\left(x-\frac{2 l \pi}{r}\right)^{-\alpha}\left|\sum_{k=n+1}^{\infty} b_{k} \sin k x\right|^{s} d x \\
& \ll \sum_{n=r}^{\infty} n^{\alpha} \int_{2 l \pi / r+\pi /(n+1)}^{2 l \pi / r+\pi / n} \mid \sum_{d=0}^{\infty}\left(\sum_{k=2^{d+1}(n+1)}^{2^{d+1}(n+1)-1+r} b_{k} \widetilde{D}_{k,-r}(x)\right. \\
& \left.-\sum_{k=2^{d}(n+1)}^{2^{d}(n+1)+r-1} b_{k} \tilde{D}_{k,-r}(x)-\sum_{k=2^{d}(n+1)}^{2^{d+1}(n+1)-1} \triangle_{r} b_{k} \tilde{D}_{k, r}(x)\right)\left.\right|^{s} d x \\
& \leq \sum_{n=r}^{\infty} n^{\alpha} \int_{2 l \pi / r+\pi /(n+1)}^{2 l \pi / r+\pi / n} \frac{1}{(r x / \pi-2 l)^{s}} \\
& \times\left(\sum_{d=0}^{\infty}\left(\sum_{k=2^{d+1}(n+1)}^{2^{d+1}(n+1)-1+r}\left|b_{k}\right|+\sum_{k=2^{d}(n+1)}^{2^{d}(n+1)+r-1}\left|b_{k}\right|+\sum_{k=2^{d}(n+1)}^{2^{d+1}(n+1)-1}\left|\triangle_{r} b_{k}\right|\right)\right)^{s} d x \\
& \ll \sum_{n=r}^{\infty} n^{\alpha+s-2}\left(\sum_{d=0}^{\infty}\left(\sum_{k=2^{d+1}(n+1)}^{2^{d+1}(n+1)-1+r}\left|b_{k}\right|+\sum_{k=2^{d}(n+1)}^{2^{d}(n+1)+r-1}\left|b_{k}\right|+\sum_{k=2^{d}(n+1)}^{2^{d+1}(n+1)-1}\left|\triangle_{r} b_{k}\right|\right)\right)^{s} .
\end{aligned}
$$


Further by Hölder inequality with $p>1$, we get

$$
\begin{aligned}
I_{2} \ll & \sum_{n=r}^{\infty} n^{\alpha+s-2}\left(\sum _ { d = 0 } ^ { \infty } \left[\left(\sum_{k=2^{d}(n+1)}^{2^{d+1}(n+1)-1}\left|\triangle_{r} b_{k}\right|^{p}\right)^{\frac{1}{p}}\left(\sum_{k=2^{d}(n+1)}^{2^{d+1}(n+1)-1} 1\right)^{1-\frac{1}{p}}\right.\right. \\
& \left.\left.+\sum_{k=2^{d+1}(n+1)}^{2^{d+1}(n+1)-1+r}\left|b_{k}\right|+\sum_{k=2^{d}(n+1)}^{2^{d}(n+1)+r-1}\left|b_{k}\right|\right]\right)^{s} \\
\leq & \sum_{n=r}^{\infty} n^{\alpha+s-2}\left(\sum _ { d = 0 } ^ { \infty } \left[\left(\sum_{k=2^{d}(n+1)}^{2^{d+1}(n+1)-1}\left|\triangle_{r} b_{k}\right|^{p}\right)^{\frac{1}{p}}\left(2^{d}(n+1)\right)^{1-\frac{1}{p}}\right.\right. \\
& \left.\left.\left.+\sum_{k=2^{d+1}(n+1)}^{2^{d}(n+1)+r-1}\right] b_{k}\left|+\sum_{k=2^{d}(n+1)}^{s+1)-1+r}\right| b_{k} \mid\right]\right)^{s}
\end{aligned}
$$

Applying Lemma 5, we have

$$
I_{2} \ll \sum_{n=r}^{\infty} n^{\alpha+s-2}\left(\sum_{d=0}^{\infty}\left[\left(2^{d}(n+1)\right)^{1-\frac{1}{p}}\left(2^{d}(n+1)\right)^{\theta-1} \sum_{k=\left[\frac{2^{d}(n+1)}{c}\right]}^{\infty} \frac{\left|b_{k}\right|}{k^{\theta}}+\sum_{k=2^{d}(n+1)}^{2^{d}(n+1)+r-1}\left|b_{k}\right|\right]\right)^{s} .
$$

From Lemma 6, we get

$$
\begin{aligned}
I_{2} \ll & \sum_{n=r}^{\infty} n^{\alpha+s-2}\left(\sum _ { d = 0 } ^ { \infty } \left[\left(2^{d}(n+1)\right)^{\theta-\frac{1}{p}} \sum_{k=\left[\frac{2^{d}(n+1)}{c}\right]}^{\infty} \frac{\left|b_{k}\right|}{k^{\theta}}\right.\right. \\
& \left.\left.+\frac{1}{1-2^{\theta-\frac{1}{p}}}\left(2^{d}(n+1)\right)^{\theta-\frac{1}{p}} \sum_{k=\left[\frac{2^{d}(n+1)}{c}\right]}^{\infty} \frac{\left|b_{k}\right|}{k^{\theta}}\right]\right)^{s} \\
\ll & \sum_{n=r}^{\infty} n^{\alpha+s-2+\theta s-\frac{s}{p}}\left(\sum_{d=0}^{\infty}\left(2^{d}\right)^{\theta-\frac{1}{p}} \sum_{k=\left[\frac{2^{d}(n+1)}{c}\right]}^{\infty} \frac{\left|b_{k}\right|}{k^{\theta}}\right)^{s} .
\end{aligned}
$$

If $\theta-\frac{1}{p}<0$, then

$$
\begin{aligned}
I_{2} & \ll \sum_{n=r}^{\infty} n^{\alpha+s-2+\theta s-\frac{s}{p}}\left(\sum_{k=\left[\frac{n+1}{c}\right]}^{\infty} \frac{\left|b_{k}\right|}{k^{\theta}}\right)^{s} \\
& \ll \sum_{n=r}^{\infty} n^{\alpha+s-2-\frac{s}{p}+\theta s}\left(\sum_{k=\left[\frac{n}{c}\right]}^{n} \frac{\left|b_{k}\right|}{k^{\theta}}\right)^{s}+\sum_{n=r}^{\infty} n^{\alpha+s-2-\frac{s}{p}+\theta s}\left(\sum_{k=n}^{\infty} \frac{\left|b_{k}\right|}{k^{\theta}}\right)^{s} \\
& \leq \sum_{n=1}^{\infty} n^{\alpha-2-\frac{s}{p}}\left(\sum_{k=1}^{n} k\left|b_{k}\right|\right)^{s}+\sum_{n=1}^{\infty} n^{\alpha+s-2-\frac{s}{p}+\theta s}\left(\sum_{k=n}^{\infty} \frac{\left|b_{k}\right|}{k^{\theta}}\right)^{s} .
\end{aligned}
$$


Now, we use Lemma 2 and get

$$
\begin{aligned}
I_{2} \ll & \sum_{n=1}^{\infty}\left(n^{\alpha-2-\frac{s}{p}}\right)^{1-s}\left(n\left|b_{n}\right|\right)^{s}\left(\sum_{k=n}^{\infty} k^{\alpha-2-\frac{s}{p}}\right)^{s} \\
& +\sum_{n=1}^{\infty}\left(n^{\alpha+s-2-\frac{s}{p}+\theta s}\right)^{1-s}\left(\frac{\left|b_{n}\right|}{n^{\theta}}\right)^{s}\left(\sum_{k=1}^{n} k^{\alpha+s-2-\frac{s}{p}+\theta s}\right)^{s} .
\end{aligned}
$$

For $1+\frac{s}{p}-\theta s-s<\alpha<1+\frac{s}{p}$, we have

$$
I_{2} \ll \sum_{n=1}^{\infty} n^{\alpha-2-\frac{s}{p}+2 s}\left|b_{n}\right|^{s}
$$

Now, we estimate the following integral:

$$
\begin{aligned}
\int_{\frac{2 l \pi}{r}+\frac{\pi}{r}}^{\frac{2(l+1) \pi}{r}} \omega_{\alpha, r}(x)\left|\sum_{k=1}^{\infty} b_{k} \sin k x\right|^{s} d x \ll & \int_{\frac{2(l+1) \pi}{r}-\frac{\pi}{r}}^{\frac{2(l+1) \pi}{r}} \omega_{\alpha, r}(x)\left|\sum_{k=1}^{n} b_{k} \sin k x\right|^{s} d x \\
& +\int_{\frac{2(l+1) \pi}{r}-\frac{\pi}{r}}^{\frac{2(l+1) \pi}{r}} \omega_{\alpha, r}(x)\left|\sum_{k=n+1}^{\infty} b_{k} \sin k x\right|^{s} d x \\
:= & I_{3}+I_{4} .
\end{aligned}
$$

By Lemma 2, for $\alpha<1$, we have

$$
\begin{aligned}
I_{3} & =\sum_{n=r}^{\infty} \int_{\frac{2(l+1) \pi}{r}-\frac{\pi}{n}}^{\frac{2(l+1) \pi}{r}-\frac{\pi}{n+1}}\left(\frac{2(l+1) \pi}{r}-x\right)^{-\alpha}\left|\sum_{k=1}^{n} b_{k} \sin k x\right|^{s} d x \\
& \ll \sum_{n=1}^{\infty} n^{\alpha-2}\left(\sum_{k=1}^{n}\left|b_{k}\right|\right)^{s} \\
& \ll \sum_{n=1}^{\infty} n^{\alpha+s-2}\left|b_{n}\right|^{s} \leq \sum_{n=1}^{\infty} n^{\alpha-2-\frac{s}{p}+2 s}\left|b_{n}\right|^{s} .
\end{aligned}
$$

Using Lemma 1 with $m \rightarrow \infty$ and the inequality

$$
2(l+1)-\frac{r}{\pi} x \leq\left|\sin \frac{r x}{2}\right| \quad \text { for } x \in\left(\frac{(2 l+1) \pi}{r}, \frac{2(l+1) \pi}{r}\right),
$$

we have

$$
I_{4}=\sum_{n=r}^{\infty} \int_{\frac{2(l+1) \pi}{r}-\frac{\pi}{n}}^{\frac{2(l+1) \pi}{r}-\frac{\pi}{n+1}}\left(\frac{2(l+1) \pi}{r}-x\right)^{-\alpha}\left|\sum_{k=n+1}^{\infty} b_{k} \sin k x\right|^{s} d x,
$$

and similarly as in the case $I_{2}$ we obtain

$$
I_{4} \ll \sum_{n=1}^{\infty} n^{\alpha-2-\frac{s}{p}+2 s}\left|b_{n}\right|^{s}
$$


Szal and Kubiak Journal of Inequalities and Applications

(2019) 2019:275

Page 15 of 18

Finally, combining (3)-(6), we obtain that

$$
\int_{-\pi}^{\pi} \omega_{\alpha, r}(x)|g(x)|^{s} d x \leq C \sum_{n=1}^{\infty} n^{\alpha-2-\frac{s}{p}+2 s}\left|b_{n}\right|^{s} .
$$

The case when $\phi(x)=\sum_{k=1}^{\infty} b_{k} \cos k x$ can be proved similarly.

4.5 Proof of Theorem 7

We prove the theorem for the case where $\phi(x)=\sum_{k=1}^{\infty} b_{k} \sin k x$. We follow the method adopted by Tikhonov [9]. Note that if $1-\theta s<\alpha<1+s$, then $\phi \in L^{1}$. Namely, if $s>1$ then using Hölder inequality, we have

$$
\begin{aligned}
\int_{0}^{\pi}|\phi(x)| d x & =\int_{0}^{\pi}\left(\omega_{\alpha, r}(x)\right)^{\frac{1}{s}}|\phi(x)|\left(\frac{1}{\omega_{\alpha, r}(x)}\right)^{\frac{1}{s}} d x \\
& \leq\left(\int_{0}^{\pi} \omega_{\alpha, r}(x)|\phi(x)|^{s} d x\right)^{\frac{1}{s}}\left(\int_{0}^{\pi}\left(\omega_{\alpha, r}(x)^{-\frac{1}{s}}\right)^{\frac{1}{1-\frac{1}{s}}} d x\right)^{1-\frac{1}{s}} .
\end{aligned}
$$

We will show that $\int_{0}^{\pi}\left(\omega_{\alpha, r}(x)\right)^{-\frac{1}{s-1}} d x<\infty$. We can write

$$
\int_{0}^{\pi}\left(\omega_{\alpha, r}(x)\right)^{-\frac{1}{s-1}} d x=\sum_{l=0}^{\left[\frac{r}{2}\right]}\left(\int_{\frac{2 l \pi}{r}}^{\frac{(2 l+1) \pi}{r}}\left(x-\frac{2 l \pi}{r}\right)^{\frac{\alpha}{s-1}} d x+\int_{\frac{(2 l+1) \pi}{r}}^{\frac{2(l+1) \pi}{r}}\left(\frac{2(l+1) \pi}{r}-x\right)^{\frac{\alpha}{s-1}} d x\right),
$$

when $r$ is an even number, and

$$
\begin{aligned}
& \int_{0}^{\pi}\left(\omega_{\alpha, r}(x)\right)^{-\frac{1}{s-1}} d x \\
& =\sum_{l=0}^{\left[\frac{r}{2}\right]} \int_{\frac{2 l \pi}{r}}^{\frac{(2 l+1) \pi}{r}}\left(x-\frac{2 l \pi}{r}\right)^{\frac{\alpha}{s-1}} d x+\sum_{l=0}^{\left[\frac{r}{2}\right]-1} \int_{\frac{(2 l+1) \pi}{r}}^{\frac{2(l+1) \pi}{r}}\left(\frac{2(l+1) \pi}{r}-x\right)^{\frac{\alpha}{s-1}} d x,
\end{aligned}
$$

when $r$ is an odd number.

Using integration by substitution, we get

$$
\begin{aligned}
\int_{0}^{\pi}\left(\omega_{\alpha, r}(x)\right)^{-\frac{1}{s-1}} d x & =\sum_{l=0}^{\left[\frac{r}{2}\right]}\left(\int_{0}^{\frac{\pi}{r}} y^{\frac{\alpha}{s-1}} d y+\int_{0}^{\frac{\pi}{r}} y^{\frac{\alpha}{s-1}} d y\right) \\
& =2\left(\left[\frac{r}{2}\right]+1\right) \frac{s-1}{\alpha+s-1}\left(\frac{\pi}{r}\right)^{\frac{\alpha+s-1}{s-1}}
\end{aligned}
$$

when $r$ is an even number, and

$$
\begin{aligned}
\int_{0}^{\pi}\left(\omega_{\alpha, r}(x)\right)^{-\frac{1}{s-1}} d x & =\sum_{l=0}^{\left[\frac{r}{2}\right]} \int_{0}^{\frac{\pi}{r}} y^{\frac{\alpha}{s-1}} d y+\sum_{l=0}^{\left[\frac{r}{2}\right]-1} \int_{0}^{\frac{\pi}{r}} y^{\frac{\alpha}{s-1}} d x \\
& =\left(2\left[\frac{r}{2}\right]+1\right) \frac{s-1}{\alpha+s-1}\left(\frac{\pi}{r}\right)^{\frac{\alpha+s-1}{s-1}},
\end{aligned}
$$

when $r$ is an odd number. 


$$
\begin{aligned}
& \text { If } s=1 \text { then } \alpha>0 \text { and } \\
& \qquad \begin{aligned}
\int_{0}^{\pi}|\phi(x)| d x & =\int_{0}^{\pi} \omega_{\alpha, r}(x)|\phi(x)| \frac{1}{\omega_{\alpha, r}(x)} d x \\
& \leq \sup _{x} \frac{1}{\omega_{\alpha, r}(x)} \int_{0}^{\pi} \omega_{\alpha, r}(x)|\phi(x)| d x=\left(\frac{\pi}{r}\right)^{\alpha} \int_{0}^{\pi} \omega_{\alpha, r}(x)|\phi(x)| d x .
\end{aligned}
\end{aligned}
$$

Further, integrating $\phi$, we have

$$
F(x):=\int_{0}^{x} \phi(t) d t=\sum_{n=1}^{\infty} \frac{b_{n}}{n}(1-\cos n x)=2 \sum_{n=1}^{\infty} \frac{b_{n}}{n} \sin ^{2} \frac{n x}{2},
$$

and consequently,

$$
F\left(\frac{\pi}{k}\right) \geq \sum_{n=[k / 2]}^{k} \frac{b_{n}}{n}
$$

Since $\left(b_{n}\right) \in G M\left(p,{ }_{3} \beta(\theta), r\right)$ and using Lemma 4 , we get for $\theta-\frac{1}{p}<0$ that

$$
\begin{aligned}
& b_{v} \leq \sum_{k=v}^{v+r-1} b_{l}=\sum_{d=0}^{\infty} \sum_{k=2^{d_{v}}}^{2^{d+1} v-1}\left|\triangle_{r} b_{k}\right| \leq \sum_{d=0}^{\infty} 2^{d} v\left[\frac{1}{2^{d} v} \sum_{k=2^{d_{v}}}^{2^{d+1} v-1}\left|\triangle_{r} b_{k}\right|^{p}\right]^{\frac{1}{p}} \\
& \leq C \sum_{d=0}^{\infty}\left(2^{d} v\right)^{\theta-\frac{1}{p}} \sum_{k=\left[\frac{2 d_{v}}{c}\right]}^{\infty} \frac{b_{k}}{k^{\theta}} \leq C v^{\theta-\frac{1}{p}} \sum_{d=0}^{\infty}\left(2^{\theta-\frac{1}{p}}\right)^{d} \sum_{k=\left[\frac{v}{c}\right]}^{\infty} \frac{b_{k}}{k^{\theta}} \\
& \leq \frac{1}{1-2^{\theta-\frac{1}{p}}} C v^{\theta-\frac{1}{p}} \sum_{k=\left[\frac{v}{c}\right]}^{\infty} \frac{b_{k}}{k^{\theta}} \ll v^{\theta-\frac{1}{p}} \sum_{k=\left[\frac{v}{c}\right]}^{\infty} \frac{b_{k}}{k^{\theta}} \leq C v^{\theta-\frac{1}{p}} \sum_{d=0}^{\infty}\left(2^{d+1}\left[\frac{v}{c}\right]\right)^{1-\theta} \sum_{k=2^{d}\left[\frac{v}{c}\right]}^{2^{d+1}\left[\frac{v}{c}\right]} \frac{b_{k}}{k}
\end{aligned}
$$

Using (7) yields

$$
\begin{aligned}
b_{v} & \ll v^{\theta-\frac{1}{p}} \sum_{d=0}^{\infty}\left(2^{d}\left[\frac{v}{c}\right]\right)^{1-\theta} F\left(\frac{\pi}{2^{d+1}\left[\frac{v}{c}\right]}\right) \ll v^{\theta-\frac{1}{p}} \sum_{d=0}^{\infty}\left(2^{d}\left[\frac{v}{c}\right]\right)^{-\theta^{2^{d+1}\left[\frac{v}{c}\right]-1}} \sum_{k=2^{d}\left[\frac{v}{c}\right]} F\left(\frac{\pi}{k}\right) \\
& \ll v^{\theta-\frac{1}{p}} \sum_{k=\left[\frac{v}{c}\right]}^{\infty} \frac{1}{k^{\theta}} F\left(\frac{\pi}{k}\right) .
\end{aligned}
$$

Elementary calculations give

$$
\begin{aligned}
\sum_{k=1}^{\infty} k^{\alpha-2+\frac{s}{p}} b_{k}^{s} & \ll \sum_{k=1}^{\infty} k^{\alpha-2+\frac{s}{p}+\left(\theta-\frac{1}{p}\right) s}\left(\sum_{v=\left[\frac{k}{c}\right]}^{\infty} \frac{1}{v^{\theta}} F\left(\frac{\pi}{v}\right)\right)^{s} \\
& \ll \sum_{k=1}^{\infty} k^{\alpha-2}\left(\sum_{v=\left[\frac{k}{c}\right]}^{k} F\left(\frac{\pi}{v}\right)\right)^{s}+\sum_{k=1}^{\infty} k^{\alpha-2+\theta s}\left(\sum_{\nu=k}^{\infty} \frac{1}{v^{\theta}} F\left(\frac{\pi}{v}\right)\right)^{s} \\
& \ll \sum_{k=1}^{\infty} k^{\alpha-2-s}\left(\sum_{v=\left[\frac{k}{c}\right]}^{k} v F\left(\frac{\pi}{v}\right)\right)^{s}+\sum_{k=1}^{\infty} k^{\alpha-2+\theta s}\left(\sum_{\nu=k}^{\infty} \frac{1}{v^{\theta}} F\left(\frac{\pi}{v}\right)\right)^{s} .
\end{aligned}
$$


Using Lemma 2, for $1-\theta s<\alpha<1+s$, we have

$$
\sum_{k=1}^{\infty} k^{\alpha-2-s}\left(\sum_{v=\left[\frac{k}{c}\right]}^{k} v F\left(\frac{\pi}{v}\right)\right)^{s} \ll \sum_{k=1}^{\infty} k^{(\alpha-2-s)(1-s)}\left(k F\left(\frac{\pi}{k}\right)\right)^{s}\left(\sum_{\nu=k}^{\infty} v^{\alpha-2-s}\right)^{s}
$$

and

$$
\sum_{k=1}^{\infty} k^{\alpha-2+\theta s}\left(\sum_{\nu=k}^{\infty} \frac{1}{v^{\theta}} F\left(\frac{\pi}{v}\right)\right)^{s} \ll \sum_{k=1}^{\infty} k^{(\alpha-2+\theta s)(1-s)}\left(\frac{1}{k^{\theta}} F\left(\frac{\pi}{k}\right)\right)^{s}\left(\sum_{\nu=1}^{k} v^{\alpha-2+\theta s}\right)^{s} .
$$

Therefore, for $1-\theta s<\alpha<1+s$, we get

$$
\sum_{k=1}^{\infty} k^{\alpha-2+\frac{s}{p}} b_{k}^{s} \ll \sum_{k=1}^{\infty} k^{\alpha-2+s}\left(F\left(\frac{\pi}{k}\right)\right)^{s}
$$

Denoting by $d_{v}:=\int_{\frac{\pi}{v+1}}^{\frac{\pi}{v}}|\phi(x)| d x$, we get

$$
\sum_{k=1}^{\infty} k^{\alpha-2+\frac{s}{p}} b_{k}^{s} \ll \sum_{k=1}^{\infty} k^{\alpha-2+s}\left(\sum_{v=k}^{\infty} d_{v}\right)^{s}
$$

By Lemma 2, for $\alpha>1-s$, we obtain

$$
\begin{aligned}
\sum_{k=1}^{\infty} k^{\alpha-2+s}\left(\sum_{\nu=k}^{\infty} d_{v}\right)^{s} & \ll \sum_{k=1}^{\infty} k^{(\alpha-2+s)(1-s)} d_{k}^{s}\left(\sum_{\nu=1}^{k} v^{\alpha-2+s}\right)^{s} \\
& \ll \sum_{k=1}^{\infty} k^{(\alpha-2+s)(1-s)} k^{(\alpha-2+s+1) s} d_{k}^{s}=\sum_{k=1}^{\infty} k^{\alpha-2+2 s} d_{k}^{s} .
\end{aligned}
$$

Applying Hölder inequality when $s>1$, we have

$$
d_{k}^{s} \ll \frac{1}{k^{2(s-1)}} \int_{\frac{\pi}{(k+1)}}^{\frac{\pi}{k}}|\phi(x)|^{s} d x
$$

Finally, using the latter estimate, we get

$$
\begin{aligned}
\sum_{k=1}^{\infty} k^{\alpha-2+\frac{s}{p}} b_{k}^{s} & \ll \sum_{k=1}^{\infty} k^{\alpha-2+2 s} d_{k}^{s} \\
& \leq \sum_{k=1}^{r} k^{\alpha-2+2 s}\left(\int_{\frac{\pi}{(k+1)}}^{\frac{\pi}{k}}|\phi(x)| d x\right)^{s}+\sum_{k=r}^{\infty} k^{\alpha} \int_{\frac{\pi}{(k+1)}}^{\frac{\pi}{k}}|\phi(x)|^{s} d x \\
& \ll\left(\int_{0}^{\pi}|\phi(x)| d x\right)^{s}+\sum_{k=r}^{\infty} \int_{\frac{\pi}{k+1}}^{\frac{\pi}{k}} x^{-\alpha}|\phi(x)|^{s} d x \\
& \leq\left(\int_{0}^{\pi}|\phi(x)| d x\right)^{s}+\int_{0}^{\pi} \omega_{\alpha, r}(x)|\phi(x)|^{p} d x<\infty .
\end{aligned}
$$

The case when $\phi(x)=\sum_{k=1}^{\infty} b_{k} \cos k x$ can by proved similarly. 


\section{Conclusions}

We have introduced two new classes of $p$-bounded variation sequences, $\overline{G M}(p, \beta, r)$ and $G M(p, \beta, r)$, where $\beta:=\left(\beta_{n}\right)$ is a nonnegative sequence, $p$ a positive real number, $r \in \mathbb{N}$, $\theta \in(0,1]$. Moreover, we have studied properties of such classes and obtained a sufficient and necessary condition for weighted integrability of functions defined by trigonometric series with coefficients belonging to these classes. In particular, from our theorems we derive all related earlier results.

\section{Acknowledgements}

The publication costs of this article were covered by University of Zielona Góra.

\section{Funding}

Not applicable.

Availability of data and materials

Not applicable.

Competing interests

The authors declare that they have no competing interests.

\section{Authors' contributions}

The study was carried out in collaboration with equal responsibility. All authors read and approved the final manuscript.

\section{Publisher's Note}

Springer Nature remains neutral with regard to jurisdictional claims in published maps and institutional affiliations.

Received: 16 May 2019 Accepted: 16 October 2019 Published online: 26 October 2019

\section{References}

1. Boas, R.P. Jr.: Integrability Theorems for Trigonometric Transforms. Springer, New York (1967)

2. Chen, Y.M.: On the integrability of functions defined by trigonometrical series. Math. Z. 66, 9-12 (1956)

3. Dyachenko, M., Tikhonov, S.: Integrability and continuity of functions represented by trigonometric series: coefficients criteria. Stud. Math. 193(3), 285-306 (2009)

4. Hardy, G.H., Littlewood, J.E., Pólya, G.: Inequalities. Cambridge University Press, Cambridge (1934)

5. Heywood, P.: On the integrability of functions defined by trigonometric series. Quart. J. Math. 5, 71-76 (1954)

6. Leindler, L.: Further sharpening of inequalities of Hardy and Littlewood. Acta Sci. Math. 54, $285-289$ (1990)

7. Leindler, L.: A new class of numerical sequences and its applications to sine and cosine series. Anal. Math. 28, 279-286 (2002)

8. Liflyand, E., Tikhonov, S.: A concept of general monotonicity and applications. Math. Nachr. 284(8-9), 1083-1098 (2011)

9. Szal, B.: On weighted $L^{p}$ integrability of functions defined by trigonometric series. J. Inequal. Appl. 2010, 1 (2010)

10. Szal, B.: A new class of numerical sequences and its applications to uniform convergence of sine series. Math. Nachr. 284(14-15), 1985-2002 (2011)

11. Szal, B.: On L-convergence of trigonometric series. J. Math. Anal. Appl. 373, 449-463 (2011)

12. Tikhonov, S.: Trigonometric series with general monotone coefficients. J. Math. Anal. Appl. 326, 721-735 (2007)

13. You, D.S., Zhou, P., Zhou, S.P.: On $L^{p}$ integrability and convergence of trigonometric series. Stud. Math. 182(3), 215-226 (2007)

14. Zygmund, A.: Trigonometric Series. Cambridge University Press, Cambridge (1977)

\section{Submit your manuscript to a SpringerOpen ${ }^{\circ}$ journal and benefit from:}

- Convenient online submission

- Rigorous peer review

- Open access: articles freely available online

- High visibility within the field

- Retaining the copyright to your article 